\title{
European Extremely Large Telescope: Cross-calibration of DIMM Monitors at Aklim Site
}

\author{
M. Sabil \\ ${ }^{1}$ LPHEA laboratory, Oukaimeden Observatory, \\ Cadi Ayyad University FSSM, \\ Av. Prince My Abdellah, BP 2390 Marrakech, Morocco
}

\author{
Z. Benkhaldoun \\ ${ }^{1}$ LPHEA laboratory, Oukaimeden Observatory, \\ Cadi Ayyad University FSSM, \\ Av. Prince My Abdellah, BP 2390 Marrakech, Morocco
}

\author{
A. Habib \\ ${ }^{1}$ LPHEA laboratory, Oukaimeden Observatory, \\ Cadi Ayyad University FSSM, \\ Av. Prince My Abdellah, BP 2390 Marrakech, Morocco \\ 2 CRMEF, Av. Mouzdalifa, BP 797 Marrakech, Morocco
}

\begin{abstract}
Differential Image Motion Monitor (DIMM) is the standard instrument for site testing, it has become the instrument of choice to measure the seeing and it is now regularly used in many observatories. Since the DIMM instruments are not identical, a cross-calibration is necessary before making a reliable comparison between any two sites using this instrument. The differences are generally due to the instrumental configuration and data processing. in order to carry out a prospecting campaign at Aklim site in Moroccan Anti-Atlas; it's one site from four selected sites to harbor the European Extremely Large Telescope (E-ELT) project.

To begin a preliminary survey campaign of measurements, a local DIMM instrument a local DIM instrument has been built, and to characterize this instrument, we have carried out a crosscalibration campaign between this local DIMM named LPHEADIMM (DIMM of Laboratory Physics of High Energy and Astrophysics) with a stable existing DIMM, hereafter IAC-DIMM (DIMM of Instituto de Astrofísica de Canarias). In this paper we represent the results of the 11 nights cross-calibration at Aklim site.
\end{abstract}

\section{General Terms}

E-ELT, Site testing, Aklim site

\section{Keywords}

DIMM, Data analysis, Cross-calibration

\section{INTRODUCTION}

The story of DIMMs Cross-calibration was started with Pedersen et al [1] when compared the DIMM results of standard seeing measurements obtained with a big $2.2 \mathrm{~m}$ telescopes measurements.
Vernin et al.,[2] calibrated DIMM-IAC with ESQ ${ }^{1}$-DIMM during March and April 1992 at Paranal. Ehgamberdiev, et al [3] at the cross-calibration of Maindnak DIMM with a GSM 2 Núnez et al [4] was compared two DIMMs units during 14 nights at SPM (San Pedro Martir), the first DIMM is that used at the Observatorio Astronomico Nacional at SPM and the second DIMM is a NOAO RoboDIMM unit (RoD) is that of CTIO (Cerro Tololo Inter American Observatory). Giovanelli et al.[5] was carried out an other comparison during two nights in 2000 October between ESO-DIMM-C1 and CTIO DIMM, The two devices were different in both hardware and software but the comparison between the data sets has shown a good agreement. Hach et al., [6] was carried out another cross calibrationin in July-August 2004 at Oukaimeden Observatory for DIMM and DIMMAR instruments( tow DIMMs developed in Oukaimeden Observatory).

In 2006, the European Southern Observatory launched a worldwide search for potential astronomical sites to host the E-ELT, five sites were sounded: Aklim (in Morocco), Izaña (in Spain), Macón (in Argentina), ORM (in Spain), and Ventarrones (in Chile).

Aklim site is located in the Moroccan Anti-Atlas mountains, at the geographical coordinates $30^{\circ} 7^{\prime} 39^{\prime \prime}$ North and $08^{\circ} 18^{\prime} 31^{\prime \prime}$ West. It is a large plateau, at an altitude of $2390 \mathrm{~m}$, about one hundred kilometers east of the Atlantic coast line, $160 \mathrm{~km}$ from the town of Agadir, and $480 \mathrm{~km}$ south of Marrakesh city. The exceptional astro-climatic conditions and topography of Morocco appear to be available for E-ELT project. To characterize Aklim site, we started a measurement campaign from January 2007 to July 2010. In the first year of this campaign, we used the local differential Iamge Motion Monitor (LPHEA-DIMM) to measure the seeing. An accurate calibration of DIMM is very important for site comparison. It is in this context that we carried out a calibration campaign of measurements, this campaign was at Aklim site from 20 May to 6 June 2008. This paper consists of six principals sections. Section 1 gives a brief in-

\footnotetext{
${ }^{1}$ European Southern Observatory

${ }^{2}$ Generalized Seeing Monitor
} 
troduction. In section 2 we give a theoretical summary of DIMM seeing. We describe the cross calibration campaign at Aklim site in section 3, data analysis in section 4, statistic and results in section 5 Section 6 presents the summary and conclusions.

\section{BRIEF THEORY OF DIMM SEEING}

The DIMM theory has been explained well in many papers, as Martin et al [7], Sarazin and Roddier [8], Tokovinin et al [9], Benkhaldoun et al [10].

The seeing is defined as the width at the half maximum of a star image at the focus of a telescope

$$
\epsilon_{F W H M}=0.976 \frac{\lambda}{r_{0}}
$$

where $\lambda$ is the wavelength and $r_{0}$ is the Fried parameter. The variance of the differential image motion (in $\operatorname{radian}^{2}$ ) between two pupils of diameter $\mathrm{D}$ at the distance $\mathrm{d}$ of each other (baseline) depends on the direction of measurement [11]:

$$
\begin{gathered}
\sigma_{\|}^{2} \simeq 2 \lambda^{2} r_{0}^{-5 / 3}\left(0.179 D^{-1 / 3}-0.0968 d^{-1 / 3}\right) \\
\sigma_{\perp}^{2} \simeq 2 \lambda^{2} r_{0}^{-5 / 3}\left(0.179 D^{-1 / 3}-0.145 d^{-1 / 3}\right)
\end{gathered}
$$

where $\sigma_{\|}^{2}$ and $\sigma_{\perp}^{2}$ are respectively the longitudinal and transversal variance of the distance between the two images centroids. These two equations allows us to obtain two estimates of $r_{0}$.

We note that direction-dependent sensitivity of the image motion to changes in seeing quality is given by the constant coefficients $\left(K=\frac{\sigma^{2}}{\lambda^{2} r_{o}^{-5 / 3}}\right)$ :

$$
\begin{gathered}
K_{\|}=2\left(0.179 D^{-1 / 3}-0.0968 d^{-1 / 3}\right) \\
K_{\perp}=2\left(0.179 D^{-1 / 3}-0.145 d^{-1 / 3}\right)
\end{gathered}
$$

$K_{\|}$and $K_{\perp}$ are expressed in $m^{-1 / 3}$. The seeing $\varepsilon_{F W H M}$ (in radians), so at the tow movements $\|$ and $\perp$ we have tow estimation values of seeing

$$
\begin{aligned}
& F W H M_{\|}=0.976 \lambda^{-1 / 5}\left(\frac{\sigma_{\|}^{2} \cos \gamma}{K_{\|}}\right)^{3 / 5} \\
& F W H M_{\perp}=0.976 \lambda^{-1 / 5}\left(\frac{\sigma_{\|}^{2} \cos \gamma}{K_{\perp}}\right)^{3 / 5}
\end{aligned}
$$

where $\gamma$ is the Zenith angle.

The total seeing is given by the average of $F W H M_{\|}$and $F W H M_{\perp}$ at $\lambda=0.5 \mu \mathrm{m}$.

\section{CROSS CALIBRATION CAMPAIGN}

This campaign was carried out from 20 May to 6 June 2008 at Aklim site between tow monitors named DIMM-LPHEA and DIMMIAC. The two telescopes were located near the house building close to each other, within a distance of approximately 4 meters, the DIMM-LPHEA was installed on $1.2 \mathrm{~m}$ pilar of ground level, the DIMM-IAC was installed on a tower of 7 meter, as shown in Figure 1 where the both instruments are pointed the same observed star. The technical parameters are summarized in Table 1

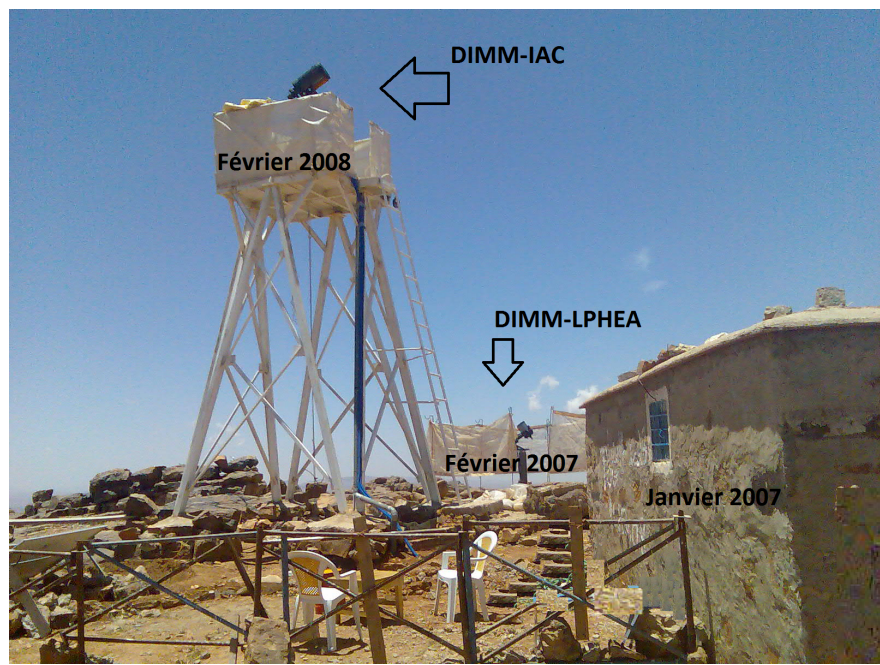

Fig. 1. Overview of the cross-calibration campaign of the two DIMMs at Aklim site

Table 1. Technical parameters of the two cross-calibration DIMMs at Aklim campaign

\begin{tabular}{|ccc|}
\hline \hline Seeing & DIMM & DIMM \\
Monitors & LPHEA & IAC \\
\hline \hline telescope Model & 8 "' Celestron & $11^{\prime \prime}$ Celestron \\
Focal distance & $2,032 \mathrm{~m}$ & $2,8 \mathrm{~m}$ \\
Diameter & $20,3 \mathrm{~cm}$ & $28 \mathrm{~cm}$ \\
Mount type & 2000 Ultima & HGM200 \\
& Fork & Losmandy \\
& (Equatorial) & \\
\hline Pupil diameter & $6 \mathrm{~cm}$ & $6 \mathrm{~cm}$ \\
Baseline & $14 \mathrm{~cm}$ & $20 \mathrm{~cm}$ \\
\hline Camera model & Webcam & PixelFly \\
& Philips & \\
toCam & 657 x495 \\
Pixel array & $640 \mathrm{x} 480$ & 0,545 \\
Pixel size & 0,568 & \\
(arcsec/pixel) & & DIMM-IAC \\
\hline Software & Seeing & \\
& DIMM-LPHEA & RG45 cable \\
\hline Port link & USB &
\end{tabular}

\section{DATA ANALYSIS}

The observations were performed during the clear nights from May 20 to June 06, 2008 at Aklim site. About 6336 values of seeing were measured during that period.

The times resolution in this cross calibration campaign was 60s, and $17 \mathrm{~s}$ at DIMM-IAC and DIMM-LPHEA respectively with the same frame $N=200$ and the same exposure time $t_{\text {exp }}=10 \mathrm{~ms}$. To obtain a good comparison of measured seeing values of the two monitors during a night, we have done a regular temporal resampling of data. The sampling period was $T_{E}=5 \mathrm{~min}$ that is greater than the temporal resolution of the two DIMMs. Thus, we compared the seeing values that are within the same temporal interval of $T_{E}$. Also and referred to eq. 6, 7, any measurement $\left(\left|\frac{\epsilon_{\perp}-\epsilon_{\|}}{\epsilon_{\perp}}\right|<0.12\right)$, are automatically rejected, Munoz-Tunon et al. [12]. 


\section{RESULTS AND DISCUSSION}

After the acquisition of data, they were analyzed and compared, we presented in figure 2 the seeing as a function of universal time obtained during the night of May 30 and June 02, 2008 where the observed star was Capella. it's the single star and can be seen. The seeing measured by the two instruments (DIMM-LPHEA and DIMM-IAC) is practically the same throughout the night.
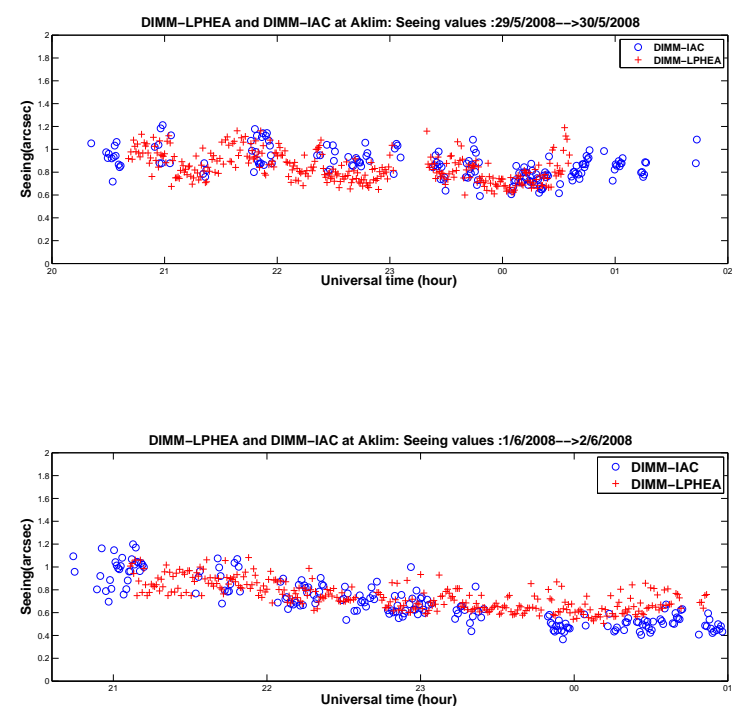

Fig. 2. Seeing as a function of universal time given by the DIMM-IAC monitor (cercal) and the DIMM-LPHEA monitor (+) at Aklim site

This figure shows clearly that the two monitors are compatible for a standard deviation $\sigma= \pm 0.1$ arcsec, also it shows that the measured values by the DIMM-IAC are overall better than the others given by DIMM-LPHEA, which may seem normal since this DIMM is installed on the tower of $7 \mathrm{~m}$, that is a less effect of the local turbulence compared to the other DIMM which is installed on a pillar of $1.20 \mathrm{~m}$.

Figure 3 shows the average nightly plot of the entire campaign at Aklim site, as well as Figure 4 presents the correlation line between between both instruments. The median and mean of DIMM-

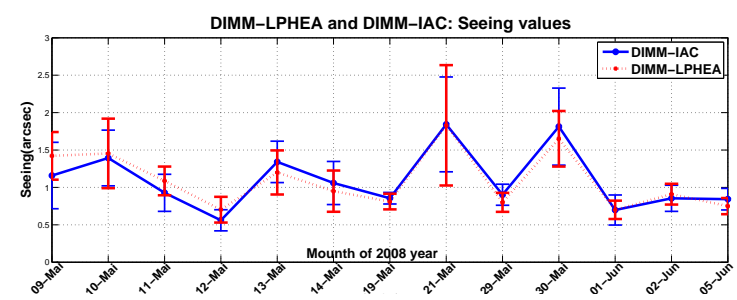

Fig. 3. Mean nightly seeing values measured by DIMM-LPHEA and DIMM-IAC of the whole cross calibration campaign at Aklim site.

IAC seeing values are $(0.92 \pm 0.31) \operatorname{arcsec}$ and $(1.09 \pm 0.31) \operatorname{arcsec}$ respectively, And those provided by DIMM-LPHEA are respectively: $(0.97 \pm 0.38)$ arcsec and $(1.10 \pm 0.38)$, The values found coincide with those obtained in Sabil et al [13] and Vernin et al [14].

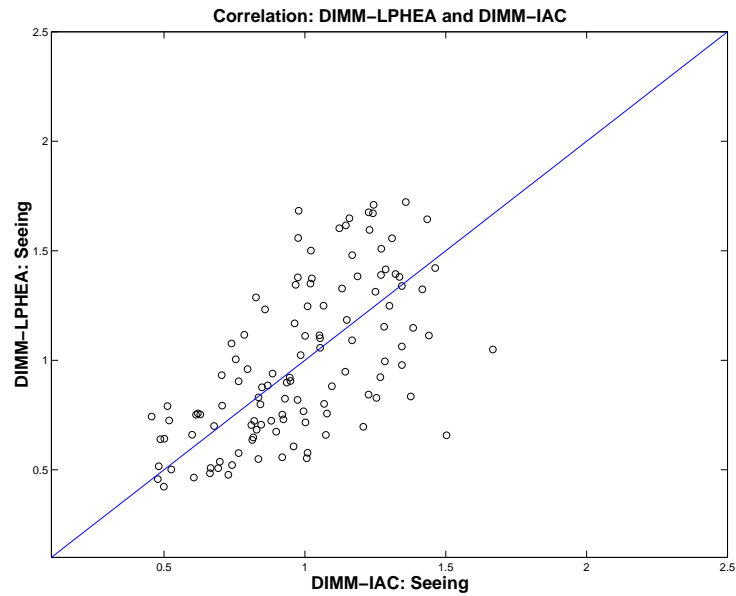

Fig. 4. Correlation between the seeing values given by DIMM-IAC monitor and that given by DIMM-LPHEA. Each point represents an average seeing over $7 \mathrm{~min}$. The correlation coefficient $=82 \%$

The correlation between measured values is shown in Figure 4 The points appear to be statistically distributed around the straight line. The correlation coefficient is the 0.82 , this indicates a perfect positive linear relationship between the two seeing values measured by DIMM-LPHEA and DIMM-IAC.

\section{CONCLUSION}

The comparisons of the main results show there exists a very good agreement between DIMM-LPHEA and DIMM-IAC measurements. The time evolution of seeing values retrieved by both exhibit highly correlated variations, showing the same main features. The number of seeing values for DIMM-LPHEA is greater than that of DIMM-IAC, because the ports link between camera$\mathrm{PC}$ are not the same, also they have a different resolution times. Since the DIMM-LPHEA gives the same results as the DIMM-IAC, and compared to the other DIMMs techniques, knowing that the DIMM-LPHEA seems to be the simplest instrument, easy to setup, and low-cost. It requires only a mask, a webcam camera and a small telescope, that makes the system easy to transport and could be very useful, also it requires less electrical energy. Thus, it is an appropriate instrument for a preliminary survey of an astronomical site, it's very reliable and cheaper compared to other DIMMs.

\section{ACKNOWLEDGMENTS}

We are grateful to the Moroccan Hassan II Academy of Science and Technology, which financially supported the site testing campaigns at the Aklim site. We acknowledge the European Community, which granted this ELT Design Study in the Framework Programme 6 (Contract 11863). It is a real pleasure to thank Jean Vernin from Nice University, Marc Sarazin from ESO and MuñozTuñón Casiana from IAC for collaboration, conversation, and advice. We provide here our sincere thanks. Our sincere gratitude also goes to the site surveyors and to all the staff members for their help and dedication. 


\section{REFERENCES}

[1] Pedersen, H., Rigaut, F., Sarazin, M.: Seeing measurements with a differential image motion Monitor. Messenger, 1988, 53, 89

[2] Vernin, J., Muñoz-Tuñón, C.: Measuring astronomical seeing: the DA/IAC DIMM, 1995, PASP 107, 265272

[3] Ehgamberdiev, S.A., Baijumanov, A.K., Ilyasov, S.P., Sarazin, M., Tillayev, Y.A., Tokovinin, A.A., Ziad, A.: The astroclimate of Maidanak Observatory in Uzbekistan. A and A Suppl. Ser. 2000, 145, 293304

[4] Núñez, J.M., Bohigas, J., Lazo, F., Hiriart, D., Calvario,T., Escoboza, O., Guillén, F.P.: Intercalibration of the San Pedro Mártir and CTIO DIMM units. Rev. Mex. Astron. Astrofis. 2007, 43, 283290

[5] Giovanelli, R., Darling, J., Sarazin, M. et al. The opti$\mathrm{cal} /$ infrared astronomical quality of high Atacama sites. I. Preliminary results of optical seeing. PASP, 2001, 113, 789802

[6] Hach, Y., Abahamid, A., Sabil, M., Benhida, A., Benkhaldoun, Z., Habib, A. and Elazhari, Y: Cross-calibration of DIMM monitors at Oukaimden observatory and Marrakesh site, Exp Astron,2010, 28:8799 DOI 10.1007/s10686-010-9192-3

[7] Martin, H. M. Image motion as a measure of seeing quality. 1987, PASP, 99, 1360

[8] Sarazin, M. and Roddier, F.The ESO differential image motion monitor. 1990, A and A, 227, 294

[9] Tokovinin, A. From Differential Image Motion to Seeing. 2002, PASP, 114, 1156

[10] Benkhaldoun, Z., Abahamid, A., El Azhari, Y. and Lazrek, M. Optical seeing monitoring at the Oukaïmeden in the Moroccan high atlas mountains: first statistics. 2005, A and A, 441, 839

[11] Sarazin, M. and Roddier, F.The ESO differential image motion monitor. 1990, A and A, 227, 294

[12] Munoz-Tunon, C., Vernin, J., and Varela, A. M. Night-time image quality at Roque de LOS Muchachos Observatory. 1997, A and A, S, 125, 183 Murdin, P. 1985, Vistas Astron., 28, 449

[13] Sabil, M., Benkhaldoun, Z., Lazrek, M., Benhida, A., Hach, Y., Habib, A. and Bounhir, A., First characterization of Jbel Aklim in Moroccan Anti-Atlas as a potential site for the E-ELT. Meteorological parameters and seeing measurements, 2010, A and $\mathrm{A}, 69,522$

[14] Vernin, J., et al. European Extremely Large Telescope Site Characterization I: Overview, 2011, PASP, 123, 1334 